\title{
MULTIAGENT SYSTEM BASED ON GENETIC ACCESS MATRIX ANALYSIS
}

\author{
Morizumi Tetsuya \\ Toyo Networks \& System Integration Co. Ltd. \\ Kawasaki 213-8584, Japan \\ moriz@olive.ocn.ne.jp \\ Suzuki Kazuhiro \\ Kanagawa University \\ Yokohama 221-8686, Japan \\ kazuhiro@kanagawa-u.ac.jp \\ Noto Masato \\ Kanagawa University \\ Yokohama 221-8686, Japan \\ noto@kanagawa-u.ac.jp \\ Kinoshita Hirotsugu \\ Kanagawa University \\ Yokohama 221-8686, Japan \\ kino@kanagawa-u.ac.jp
}

\begin{abstract}
How should an individual contribute to the public good? Conversely, how does the public help the individual? We should analyze and alleviate conflicts in community clouds. Covert channels in the access matrix are caused by conflicts between public values and a private sense of values. We cannot control the information leaks from the covert channels by using only access control. We believe that the community cloud system should emphasize harmony between public values and a private sense of values. We interpret the access matrix as follows: The acts of the individual are generalized and symbolized by an access matrix that describes the access operations of the subject. We propose a multiagent system embodying the concept of swarm intelligence to analyze the covert channels that arise. Each agent has a group target and an individual target. The group target and an individual target include targets for generation of access and restriction of access. The system does not have any principle of universal control. Instead, an agent's interactions are guided by metaheuristics for achieving targets.
\end{abstract}


The social order of the whole society is made from the agents' interactions related to the group value target, group game target, an individual value target, and an individual game target. The conceptual framework and multiagent system presented here are intended to support people. If the covert channel problem can be solved, it will become possible for people to use community clouds safely.

Keywords: Access Control, Multiagent Systems, Genetic Programming, Swarm Intelligence, Ethical Aspect

\section{INTRODUCTION}

Cloud computing has been analyzed from various aspects. In this study, the kernel of the cloud is composed of smart phones and data centers, and the aim of the cloud is to enable a market of customer resources. A problem that occurs in such a cloud is excessive concentration of customer resources. However, it is difficult to intensively manage customer resources that are meant to meet various demands. Various demands arise from conflicts between the values of the public and the individual, i.e., conflicts between notions of cooperation and competition in business. A system that collects resources will be essential in the future, and a dangerous problem is that interests of an individual may be dominated by public ones. A decentralized system could be used to control an aspect of the public and an aspect of an individual. Here, we will describe a decentralized multiagent system for the Internet available for use.

In the future, important information will be stored in a cloud of huge information resources on the Internet. We believe that people will need an agent for managing personal information. Then, the question boils down to what concepts should be used in the design of the agent system. One way of approaching this problem is to look at it in more general terms. That is, "How should society be ordered?" We know that each person has a private sense of values, and persons gather to form communities. From a person's viewpoint, he or she would call such a community "the public." Each person has such a view, and an individual who belongs to the public community try to harmonize his values in consideration of economic merits and the need to maintain social order. However, a social order is changeable and cannot be described by the universal truths of a predicate logic. In this study, therefore, we shall assume that the social order is variable and necessary to harmonize values circulating between the public and an individual. Moreover, an individual not only uses language to convey his desires; his behaviors also 
reflect his desires. The agent is a social device that only projects values of an individual through language and society. To show semantics reflecting an individual's private sense of values and the diversity of others' private senses of value, agents should be configured to support an individual. To do so, the agent should restrict the information flow and prevent information leakage and information falsification. In the future, logical agents behaving like a swarm of living things within the cloud could work on people's behavior.

We shall make the following assumptions: An agent system should evolve according to changing social order in analogy to the natural evolution of living things. Each agent is controlled by metaheuristics that encourages it to form groups with other agents, and it has targets for its actions. The targets have two aspects of conflict. The first aspect is the conflict between the public and an individual. The second aspect is between our natural desire "to win the game" and have "a good life ${ }^{1}$ with peace of mind." The agent's behavior is described by access matrices and rules governing actions. The rules and the access matrices are analogous to living things. Living things interact with one another. The agents interact by exchanging access operations and objects. The triple (a subject, an access operation, and an object) expresses the agent's behavior. A subset of the agent's behavior is defined as a gene. Moreover, the semantics of an individual evolves by exchanging subsets of the agent's behaviors in analogy to evolution in living things. In this way, access matrices evolve through exchanges between the access matrices of the group and access matrices of an individual. This approach requires agents only to interact. Note that the social order is assumed to change. Even if the changing social order is assumed, the agents should able to prevent information leakage or information falsification through covert channels. This is a safe situation for the group.

The evolutionary agent system doesn't require $a$ priori principles. That is, the agent's ability to "change" works instead of necessitating a priori principles. The system evolves in response to environmental perturbations of the community. The concepts of metaheuristics are used as a principle of evolution. Algorithms based on metaheuristics do not need to find a unique solution. This means that the system can be used to prevent information leakage from a changing cloud of the Internet that is difficult to model uniquely. 


\section{PHILOSOPHICAL FRAMEWORK FOR SOCIAL SYSTEMS}

\subsection{Philosophical Considerations}

If an individual considers himself a subject that observes the world, others are parts of his environment. Moreover, others belonging to the logical environment are viewed as others' sets of languages and others' sets of actions representing an individual's "good life and peace of mind." The environment would seem like a mirror reflecting the self. Also, an individual would seem like a mirror reflecting his "self" by using language. In this chapter, we will distinguish the self and an individual properly. In sum, the self is "I" with mind, and the individual is "I" symbolized in language.

The opposite can be said from the side of the environment. The environment entails the public, society, or others. There is a logical limit or a language limit to the ability of an individual to recognize and describe the world. Therefore, an individual does not necessarily transmit everything he or she says or thinks to others. That is, are there situations when we should stay silent? An individual and others should make an effort "to lead a good life" through and in a field of "acts of interaction." However, as mentioned above, semantics cannot completely describe this sort of world. Only interactive behaviors can express it ${ }^{2}$.

The significance of the existence of the individual values lies in the circulation of values. The world that others would recognize would be completely reversed for the individual. Such a world would not be describable with one axiomatic system. The world would be a field where the interacting entities are described by metaheuristics. That is, it might be a world of the living thing. The Internet is an autonomous, decentralized system that makes it easy to process an interaction mechanism. Therefore, it is not very difficult to introduce the agents that embody the concepts of evolving living things in the Internet.

The agents should have semantics based on the individual values. They should also reflect the diversity of the individual values. Even if an individual cannot talk about all of his values, he should not be silent. The individual should show his behavior to the society, and the agent should be the tool for this. Moreover, the language exchanges between agents should express a subset of the acts of an individual, i.e., a capability as defined above. A person's actions are described by a capability, and the set of capabilities comprises the access matrix. Capabilities are exchanged when a service is required by users. The service changes to meet the needs of the 
users, if necessary. The living things analogy fits such a situation. When the rules of the acts and the access matrices represent a living thing, this capability is equivalent to a gene.

People should not only share a common target that describes a world in semantics but could have semantics individually. The descriptive limit of semantics for an individual should be reconstructed by the evolutional interaction system. In this way, a person's interactions should recognize his own and others' existence. In other words, persons acknowledge other people's existence during their interactions. Thus, agents should take into account an individual target and a group target.

This study is based on the philosophical considerations ${ }^{2,} 3$. A circulation of values arising through linguistic exchanges between the public and the self is assumed to be necessary for a framework that makes " $a$ good life" possible. The above philosophical considerations can be summarized as follows:

(1) It is insufficient to use only language to indicate something; the semantics would not necessarily be clear to the self.

(2) If the semantics have a descriptive limit, it is difficult for the self to use them to establish the existence of the object or interpret semantics.

(3) The necessary condition for the society and the self to have a good life should not be based on sharing static states of knowledge described by the semantics.

(4) The necessary condition for a good life should instead be based on changes in the interaction. In other words, it is necessary to maintain a social order in a changing environment where the individual and the community can change.

(5) "A good life" in society is as follows: The "I" obtains a present and a non-universal solution for the self. At the same time, it interacts with others to achieve a social order. "A good life" should be a harmony of such actions.

(6) How should social order be defined? It should be defined such that it harmonizes changes in the interaction.

(7) How should the changes in the interaction be defined? The changes should be expressed in terms of the difference between the objective of the self and the neighborhood's behavior or the difference between the objective of the self and the changing social order.

\subsection{Vision for the System}

The cloud must be secure enough to prevent data leaks or falsifications. However, the cloud security should not be based on a static picture 
regarding the sharing of information but rather based on changing interactions. The algorithms that can deal with such changing interactions are metaheuristics, and the metaheuristics of the swarm intelligence are built in a multiagent system.

Our three visions for a system that can secure changing interactions are as follows:

\section{1) The world of interaction}

Logic should be interpreted from two observational aspects: the aspect of an individual and the aspect of the self. The existence of the self and others is reflected in the interactions. The objectives of an interaction are symbolized by an individual target and the group target. Our interpretation of the self, the individual, the environment, and the public is as follows:

a) The world of the self

The means of recognition of the self are projected in language or semantics. From the aspect of the self, the self could not necessarily describe all things by using language or semantics. Therefore, not everything about a self is necessarily recognized by others. That is, the self ultimately exists in a closed self-referential world. The self would try to express himself by using language or semantics.

b) An individual symbolized by language sign

An individual is an "I" defined and described in terms of language or semantics. An object controlled by the computer system directly is not the self but an individual. In other words, I appear to be an individual to people and agents in the external world, whereas I am my "self" in my mind and others are the set of individual in my mind. The difference between the self and the individual is very important for the cloud system because it is the self, not the individual, that has a sense of values (called "mental values" in what follows). And only the self in the individual can recognize the world of himself. The interpretation of the self would determine the cloud system structure.

Once an individual has been reflected in the semantics, he can be anywhere in the society of the cloud system. That is, others would be able to interpret the individual symbolized in semantics. The place of the individual in the society of the cloud is a field of games and a field of mental values. 
c) Environment of the self

The environment is the world that the self recognizes is not of itself. Others recognize the self as part of the environment. The self cannot talk about all things in the environment. However, to understand the world, "I" should have to grapple with the environment by using language and logic.

d) Public symbolized by language sign

In this study, the public is interpreted in terms of the definitions of the self, the individual, and the environment as follows. The public is an environment described in language and semantics by the self.

In other words, a place where people would be able to speak with each other is the "public." The structure of the public domain includes a game field and a mental values field. The public may experience conflicts between these fields.

\section{2) Circulation of values between the public and the individual}

The public field depends on language. The mental values of an individual are reflected in the public values and the public game. The circulation of values arises through linguistic exchanges between the public and the individual.

\section{3) Changing social order}

People try to achieve the group target of the public and an individual target. The entire order appears only as a result of the actions of the individual. In this study, such an order is called a "changing social order."

\section{METHODOLOGY}

\subsection{Security for Community Cloud}

If access authority is given to a file name, and if the content of the file leaks out through certain routes, the routes are called "covert channels." The covert channel is a topic of security modeling ${ }^{4}$. The covert channel problem is especially serious in a community cloud environment ${ }^{3,5}$ where the users who have various private senses of value are supposed to cooperate. The covert channel problem is shown in Figure 1. 


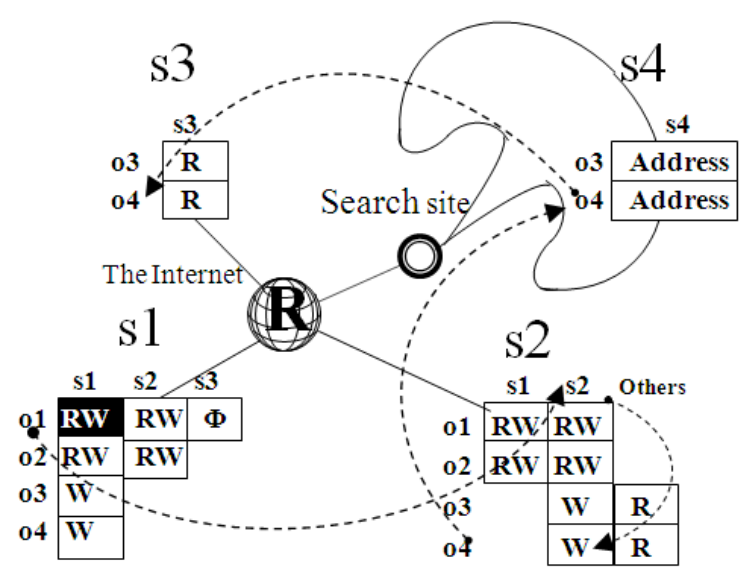

Figure 1. Example of a covert channel

Suppose that subject s1 and subject s2 are mutually federated and subject $\mathrm{s} 1$ and subject $\mathrm{s} 3$ are in a conflict of interest with each other. If subject s2 does not know the conflict relation between subject s1 and subject $\mathrm{s} 3$, we can envision the following scenario.

(1) Subject s2 reads the content of object o1 and writes it to object o4.

(2) The crawler (search robot) obtains the file name object of 04.

(3) Subject s3 searches for and finds hits for object o4 in a search engine.

(4) The content of object o1 can be accessed by subject s3, i.e., the covert channel occurs.

\subsection{Scenario for the Resolution}

To find the routes of covert channels, we should consider the following issues: When a subject must not access an object, to what extent should access authority be permitted when agents cooperate? What is the category of the existence of the object? Who should own an object? We need answers to these questions, and the answers should be reflected in the system.

There is a conflict between an act of generation of access and restriction of access actions in the access matrix, and it stems from conflicts between public values and private senses of value $e^{2,6}$. To resolve these conflicts, the agent system should support people by using logical semantics and metaheuristics, and the system should be designed to avoid conflicts between public and private values. However, a logical model (i.e., a theorem in logic) has a limited ability to describe the world, and this limitation adversely affects a computer system's ability to act as a social device. The theorems of predicate logic are described by closed formulas. Closed formulas contain universal quantifier and existential quantifier. And the law 
of the excluded middle means that logical truth is an absolute truth and interpreted as a universal. However, a logical description can only describe what is already known. But the world contains an uncertainty. Therefore in other words, in semantics, there is a limit when it comes to using logic to describe the world.

We would like to have the ability to describe what we do not know yet and describe changing social orders while acknowledging the limitations of logical descriptions and emphasizing instead an individual semantics. For this, we can exploit the concept of "swarm intelligence" as a way to "connect" an individual semantics. The conflicts between generation of access and restriction of access cannot be resolved by semantics alone but might be resolved by using "swarm intelligence."

\subsection{Evolutionary Swarm Intelligence System}

It is necessary to reflect a private sense of values in semantics to resolve conflicts between the public and the individual ${ }^{2,6}$. But there are various private senses of value. For instance, the interpretations of physical phenomena vary depending on the person. And, if people interpret the world, logical universality will be variously defined. The individual interprets their worlds by using their own semantics. But semantics are limited in their ability to describe the world, and that means that the various semantics of the individual are almost impossible to integrate. It might be an end of a big story. How should we overcome or deal with this situation? We might be able to take a hint from the behavior of flocks of birds and schools of fish. A flock's behaviors are the results of its members' interactions, and the flock does not have a leader. This is an analogy wherein the individual behaves by taking the public into consideration.

A person in a community has two kinds of target. One is an individual target that is a personal goal of some kind. The other is the target of the group to which the individual belongs. Of course, in a real society, targets may be subdivided, overlap, or be related to each other.

However, for the sake of simplicity in this study, the targets of the model will be simply a public target and an individual target. Figure 2 illustrates the swarm intelligence concept of an agent system that evolves and supports the individual. The agent's behavior is as follows:

(1) The agents that are members of the community exchange capabilities (a subset of access actions).

(2) A covert channel analysis is done to detect information leaks and falsifications caused by exchanges of capabilities. 
(3) Each agent judges the value of the covert channels and measures a mathematical distance that is conceptually defined for each target. The targets are described in semantics.

(4) Each agent continuously gathers information within a range of convergence to the targets. Metaheuristics are used to identify the targets.

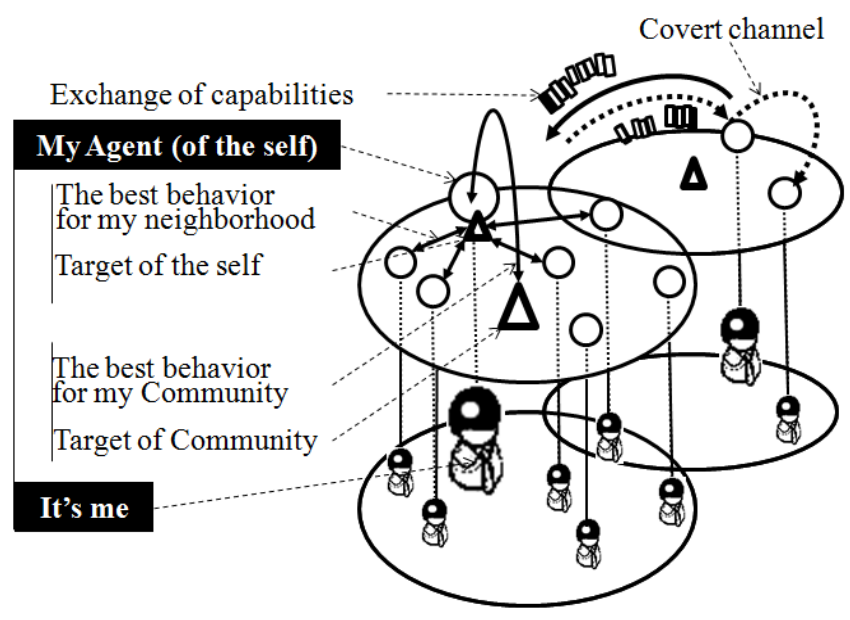

Figure 2. Swarm intelligence concept

Typical swarm intelligence models include the Boid model, the Particle Swarm Optimization (PSO) $\operatorname{model}^{7}$, the Ant Colony Optimization (ACO) model, and the Genetic Programming (GP) model.

The Boid model describes the actions and relations among members called "Boid". Swarm intelligence movement is described by an easy rule concerning interaction among Boids. However, the model has neither a concept of evolution nor a concept of convergence to a specific solution.

The PSO model provides two kinds of solutions, the local best and the global best, and a mechanism to converge them to the same value. This model is effective when a certain evaluation function is given. However, the PSO model does not include a concept of evolution. However, it is not possible to describe the evolution.

The ACO is an algorithm that calculates the best graph route stochastically. The analogy is as follows: The ants go along on the road where the pheromone was put by the ants and obtains the solution efficiently. 
The GP model is described by a metaheuristic process from which the members evolve by making changes to their "genes" by referring to an environmental rule. A gene is also considered a candidate for the solution. The actions relating members of the swarm are random in the GP model. Therefore, in this model, it is difficult to describe relations between the members in the swarm.

The model proposed in this study combines the concept of the four metaheuristic models. The access triple that describes the ability of the agent corresponds to the gene of the GP model. We call the access triple a "capability." The agent operates with the aims of achieving an individual target and a group target as in the PSO model. Each target has a strategy to try to make a swarm, and each one has a strategy to shun confrontations. The strategy to make a swarm is based on generating values and restricting confrontation. And it keeps the occurrence of covert channels at a constant rate.

\section{STRUCTURE AND BEHAVIOR OF THE SYSTEM}

\subsection{Structure of the System}

\section{1) Swarm intelligence}

The group is maintained by simple mutual actions of the agent for the individual. The swarm intelligence concept is exploited for value circulation between the public and the individual. Swarm intelligence evolves in a way that harmonizes the individual and society.

\section{2) Provision defined as a candidate target}

Swarm intelligence should be evolved. And metaheuristics are a sufficient condition to evolve. Metaheuristics are algorithms that repeatedly perturb a solution until convergence is obtained. The solution is considered the target of the actions in this system. We call the candidate target a "provision", and it is described by the access matrix and the rules of behavior of the individual or society.

The etymology of the provision is to prepare for actions. We interrupt the meaning of it as a candidate of actions. The metaheuristics of this system have four kinds of provisions. And the provisions are reflected by four targets. The targets lie beyond track of actions in access matrices to converge. The provisions are as follows: 
a) Individual values

A provision of a private sense of values includes rules of generation of access and restriction of access concerning individual values. Individual values are reflected in the access matrix of the neighborhood around the individual. Even if the agent has two targets of the individual and group, agents that influence each other are the agents defined by a specific distance. We call the distance 'neighbor'.

b) Public values

A provision of public values includes rules of generation of access and restriction of access concerning public values. Public values are reflected in the access matrix of the community (group).

c) Individual games

A provision in the private game includes rules of generation of access and restriction of access concerning individual games. The individual games are reflected in the access matrix of the game of the neighborhood around the individual.

d) Public game

A provision in the public game includes rules of generation of access and restriction of access concerning a public game. A public game is reflected in the access matrix of the community (group).

\section{3) Evolution}

a) Provisions as a set of genes

The access matrix describes the access authority of a subject to an object. In other words, the access matrix is a model that abstracts the actions of the subject upon the object. Therefore, the provision of the group symbolizes the evolving target of groups of living things, and the access matrix of the individual symbolizes the evolving target of the individual. The track of actions is defined as a set of data and behavior. And the target is defined as its own variation. Therefore evolving track of actions implies evolving target. In other words, these provisions correspond to the genes of living things. Hereditary transmissions occur through the exchange of genes representing capabilities.

b) Adaptation and selection of provisions

The components of the access matrices are changed when different provisions are selected. Detected covert channels are evaluated from the 
standpoint of public and private values. The selected actions are ones that face the individual toward the target or face the community toward the target. This is a way to express adaptation and selection of the provision. Discovery of covert channels prompts changes to the access authority and restrictions on the sets of objects and community members. In a word, the selected provision is not to the better for either the group or the individual.

c) Inheritance of genes

Individual agents exchange genes with each other. Genes are inherited. The intersection set of two agents' capabilities is kept, and capabilities that are outside the intersection set are evaluated and added to their access matrices.

\subsection{Behavior of the System and An Individual}

\section{1) Behavior of the entire system}

Figure 3 shows the interactions among the access matrices that symbolize the four provisional targets ( $\mathrm{a}, \mathrm{b}, \mathrm{c}, \mathrm{d}$ of section 4.2-2).

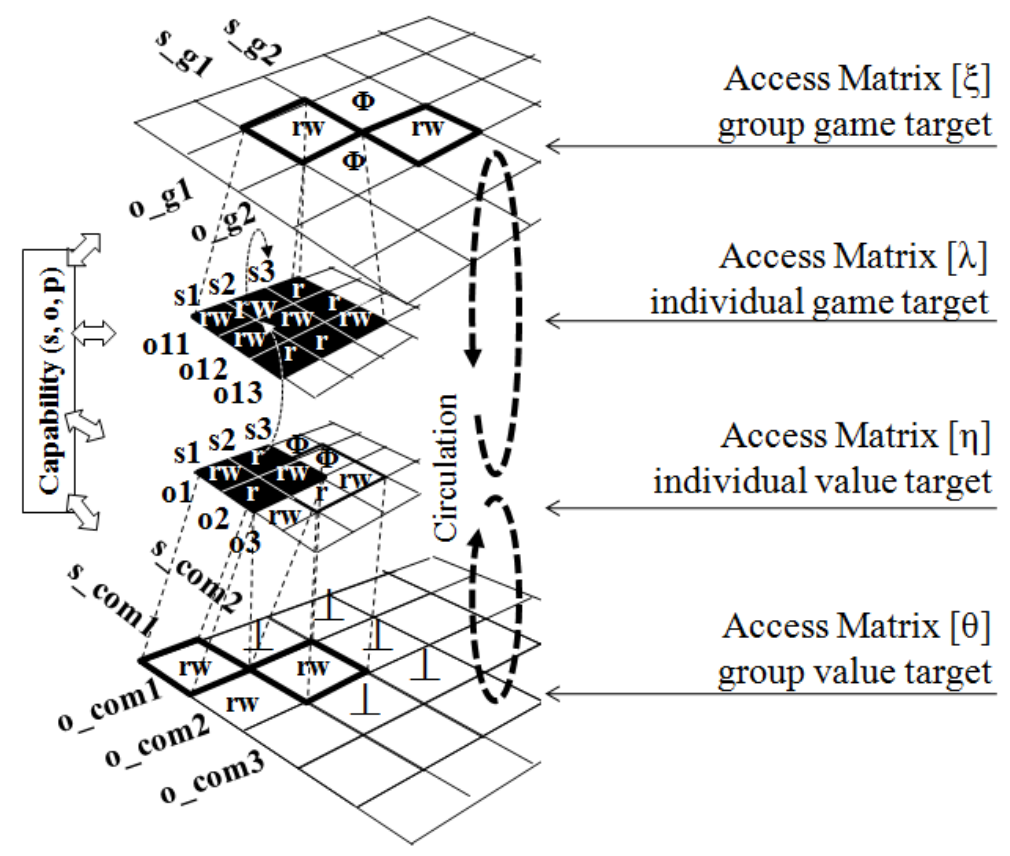

Figure 3. Value circulation among access matrices

Public and private values circulate among the access matrices (Provision). Figure 3 shows circulations between individual value target [ $\eta]$ and group value target $[\theta]$ and between individual value target $[\eta]$ and group 
game target $[\xi]$ by short dashed lines with arrows. The capability in Figure 3 is the input or output of the access matrix of the individual agents. Similarly, group and individual values about target $[\lambda]$ circulate in the individual games.

The granting of access authority is not necessarily from the group access matrix to the individual access matrix. However, it is necessary to record the conflict logs of the access matrices that arise from conflicts between the public and the individual. The stored log of conflicts becomes knowledge to identify the covert channels of the individual access matrix.

\section{2) Interactive behaviors of individual agents}

Each agent has four targets. The agent has group targets for game [ $\xi]$ and individual value $[\theta]$ and individual targets for game $[\lambda]$ and individual value [ $\eta]$. Interactions occur between people through the actions based on judgments of the game and the actions based on judgments of individual values. We think that the concept to distinguish the action by the aspect of the game and value is very important for interactions of the system. When the purpose of the system control is suitable for information security, the interactions can be represented in terms of generation of access and restriction of access. When we act, will is reflected in the action, and our will is usually symbolized by language sign. Therefore, actions concerning the games and values are reduced to symbolized language sign. In information processing, the agent must process such reduced actions. Furthermore, actions reduced to language can be classified into affirmatively symbolized events and negatively symbolized events. Affirmatively symbolized events indicate generation of access for the individual and group, whereas negatively symbolized events indicate restriction of access for the individual and group. Agents should be made to form a swarm and exclude other agents that do not conform to the order of the swarm. The behavior of agents making up the swarm conforms to the aspects of generation of access and restriction of access as follows:

(1) Group targets are those with generation of access and restriction of access within a group.

(2) Individual targets are those with generation of access and restriction of access between neighbors.

(3) The target of the generation of access is reduced to "making a group through evolution," and the target of the restriction of access is "to exclude an individual from a group" or "to keep the individual away from the center of the group." 
Figure 4 shows the actions of the four targets of agent_i at time k. Here, $[\eta]$ res is an individual restriction target, and [ $\eta] \_e m g$ is an individual generation target.

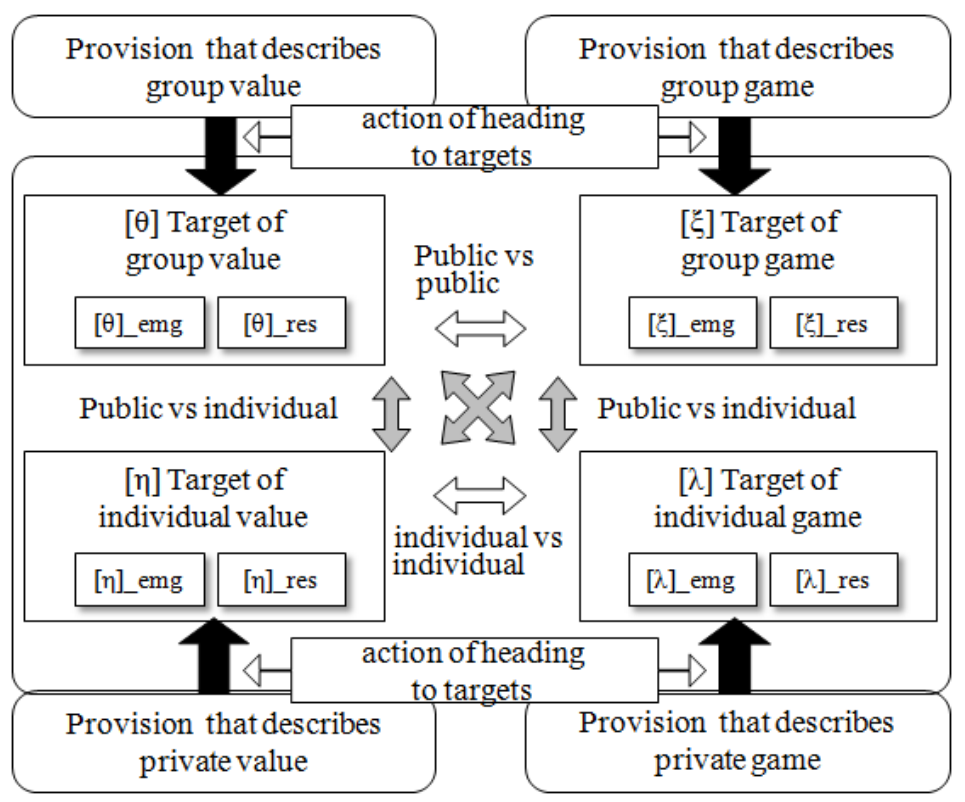

Figure 4. The actions of four targets of Agent_i at time k

There is no universal control for the agent to head toward a target. Instead, there are the metaheuristics that guide the agent toward the targets and develop through the agents' interacting. The targets are always changing. To reach the changing targets, neighbor-agent target selection rules and group target selection rules are necessary. Interactive operation between the agents would appear in the access log sequence in the access matrix of each agent. We will call the access log sequence "tracks of actions." For instance, the "tracks of action" are a sequence as follows: subject $\mathrm{s} 1$ writes object $\mathrm{o} 1$, object $\mathrm{o} 1$ is read by subject $\mathrm{s} 2$, and subject $\mathrm{s} 3$ reads object 02 and writes it down in object 03 . In other words, the "tracks of actions" are a sequence of "capabilities." The "tracks of actions" would be caused as a result of the agent's executing some services. Therefore, agents who execute the same service will have similar routes. A set of the "tracks of action" makes an access matrix. The following behaviors are aimed at achieving the target after covert channels are detected:

(1) Identify the behaviors that cause covert channels in the individual access matrix.

(2) Select the rules that show which target is that of the identified covert channels. 
(3) Enforce neighbor-agent target selection rules and target selection rules of the group to which the agent belongs.

(4) Intercept the covert channels in the following way: change access authority, permit access to the covert channel route, and add a new capability.

(5) Select the provision that results in a constant number of covert channels.

\section{DISCUSSION}

\subsection{Actions Attracted to the Swarm}

When the agent creates the swarm, the swarm has a target called a "provision". A provision is described by the access matrix. At that time, the agent moves like it is drawn by the "gravitation" of the swarm. However, the gravitation, though it is an analogy, is not an element of a static access matrix. It is composed of the tracks of actions that occur in the access matrix.

The tracks of the actions make power to attract to the swarm. The act of forming the swarm is the gravitation that attracts the person to the swarm. The gravitation appears as the tracks of actions. And the tracks of actions would be caused as a result of the agent's executing some actions (services in the Internet). The tracks of the actions resemble an ant that traces pheromone trails in the Ant Colony Optimization. An inherited selection of the swarm works on the basis of some parameters like the "tracks of actions". The targets of the agents are derived from the state of the sequence of actions and the parameters.

The PSO requires an evaluation function, and the difference between evaluation values and observed data plays an important role in finding a convergent solution. However, our system does not assume a priori evaluation functions and evaluation values. Instead, neighboring agents head to their individual targets and group target while using the function of the Boid and the ACO. The target of the swarm is described by the center of gravity of the swarm. The "tracks of actions" are the center of gravity in our model.

\subsection{Why Is Evolution Needed?}

The reason why the concept of evolution is needed is as follows:

(1) The language has a limit when it describes the world ${ }^{8}$. 
(2) Therefore, the viewpoint of "the others' eyes" will be indispensable in order to overcome the limit.

(3) Not only the relation between "I" and the others but behavior is in important positioning. The behavior of "I" and the behavior of the others will appear in "a social (i.e. swarm) rule".

(4) However, "We obey the rule blindly". That is, a rule does not exist a priori.

(5) Nevertheless, we cannot live only one person. And we wish further we would like to live "a good life" in a swarm.

(6) "I" exists between the "self" in my mind and linguistic "individual". The place is a place in which the "I" and the others do an interaction.

(7) Therefore, it is required to express and design behavior of an interaction as a tool for living "a good life". And the tool must be used by people in order to "be good life" in a swarm.

(8) However, there should not be any fixed rule for the purpose of making a society. On the other hand, a society must not collapse. Moreover, the aim set up by the members must be achieved.

(9) The necessary condition which goes to a target while fluctuating a rule toward a target is the evolution. The various values in our mind will be reflected in data that are the parameter of the evolution function.

(10) The covert channels are located in the evolving behavior.

\subsection{Philosophy of System that Is Interactive and Evolves}

The philosophy of a system with a changing objective and without $a$ priori solutions can be outlined as follows.

(1) Real existence appears in the interaction. Because we assumed that an absolute, universal solution is not required in society or a community, each individual must set its own target.

(2) An individual is a virtual self.

(3) Others are the environment of the self.

(4) To live well, the self should make decisions for himself. However, because the self lives socially, it is necessary to obtain agreement in the society as an individual.

(5) A swarm is a set of the individual.

(6) Selves make a swarm in order to live well.

(7) Selves interact with others by using the language from the standpoint of the individual. At that time, others are the environment of the self.

(8) There is not only one solution to live well in the swarm but rather objectives for doing so. 


\subsection{Implications and Benefit to the Cloud}

Three system visions shown in section 2.2 (the world of interaction, circulation of values between the public and the individual, and changing social order) are the necessary conditions for the "I" or "me" of the self to behave as an individual on the Internet. We proposed the evolving swarm intelligence from those visions. However, the current Internet does not have agents that support a person like that. The current Internet is only a system and a service provided by a very huge and almost-uniform search system. And the current Internet has the tendency to make mental values more uniform. In contrast, our system would not depend upon a system that has uniform semantics or a uniform concept. The agents would support each person or organization. The agents in the Internet should support people's linguistic activity. It is very important that people connected with the Internet can act without their senses of values ruled. Such a system will be needed so that the society can maintain various senses of values and each individual can harmonize their actions with the society.

\section{CONCLUSION}

In community clouds, information leakage and information falsification caused by covert channels cannot be controlled only through access control matrices. For new information to emerge safely on the Internet, such covert channels must be analyzed and controlled. In this study, we did not limit this problem to a specific service or system but rather proposed a generalized framework to deal with such problems. That is, the access matrix is interpreted as a symbol of the action with which the person produces the semantics. We philosophically investigated our vision of the system. We concluded that the semantics that reflect our recognition of the existence of the covert channels in the access matrix arises from the confrontation between public values and a private sense of values that exists in the society. "To have a good life," we should recognize the conflicts around us, and we should harmonize public values and a private sense of values by using the metaheuristics. The proposed system is a multiagent system with a swarm intelligence model. Each agent has its own semantics, but the interactions between the agents are the metaheuristics. Each agent has group targets and individual targets. And each target is a particular value and a particular game. A provision that describes action rules and capabilities of the agent is also a target and candidate solution. If the covert channel problem were to be solved in this way, it would become possible for people to coordinate business and study endeavors in a community cloud safely. 


\section{REFERENCES}

[1] Plato, Dover Thrift Editions, Benjamin Jowett, PLATO Six Great Dialogues: Apology, Crito, Phaedo, Phaedrus, Symposium. The Republic: Dover Publications, 2007.

[2] MORIZUMI Tetsuya, KUBO Naoya, SUZUKI Kazuhiro, and KINOSHITA Hirotsugu, Floating order concept of the cloud (Proposal of community provisioning). Technical Report of the Institute of Electronics, Information and Communication Engineers (IEICE), 110(231), p13-18, 2010.

[3] MORIZUMI Tetsuya, SUZUKI Kazuhiro, and KINOSHITA Hirotsugu, A system of search, access restriction and agent in the clouds. Paper Presented at the 9th Annual International Symposium on Applications and the Internet, Seattle, USA, July 20-24, 2009.

[4] Patrick R. Gallagher, A guide to understanding covert channel analysis of trusted systems. Retrieved March 31, 2011, from http://www.fas.org/irp/nsa/rainbow/tg030.htm.

[5] Peter Mell, and Tim Grance, NIST Definition of Cloud Computing. Retrieved March 31, 2011, from http://csrc.nist.gov/publications/nistpubs/800-145/SP800-145.pdf.

[6] KUBO Naoya, MORIZUMI Tetsuya, SUZUKI Kazuhiro, and KINOSHITA Hirotsugu, Multi agent system in order to distinguish the floating context of personalities. Paper Presented at The Institute of Electronics, Information and Communication Engineers (IEICE) the 2011 Symposium on Cryptography and Information Security, SCIS2011, Kyoto, Japan, January 25, 2011.

[7] MATSUI Takeya, NOTO Masato, and NUMAZAWA Masanobu, A hybrid particle swarm optimization considering accuracy and diversity of solutions. Paper Presented at the Systems Man and Cybernetics (SMC), 2010 IEEE International Conference, Istanbul, Turkey, October 10-13, 2010.

[8] Ludwig Wittgenstein, P.M.S. Hacker, Joachim Schulte, Philosophical Investigations. Chichester: Wiley-Blackwell, 2009. 
